\title{
Exploration of the Cell-Cycle Genes Found Within the RIKEN FANTOM2 Data Set
}

\author{
Alistair R.R. Forrest, , 2,3,7 Darrin Taylor, ${ }^{1,2,3}$ RIKEN GER Group ${ }^{4}$ and GSL \\ Members, ${ }^{5,6}$ and Sean Grimmond ${ }^{1,2}$ \\ ${ }^{1}$ The Institute for Molecular Bioscience, University of Queensland, Queensland Q4072, Australia; ${ }^{2}$ University of Queensland, \\ Queensland Q4072, Australia; ${ }^{3}$ The Australian Research Council Special Research Centre for Functional and Applied \\ Genomics, University of Queensland, Queensland Q4072, Australia; ${ }^{4}$ Laboratory for Genome Exploration Research Group, \\ RIKEN Genomic Sciences Center (GSC), RIKEN Yokohama Institute, Suehiro-cho, Tsurumi-ku, Yokohama, Kanagawa, \\ 230-0045, Japan; ${ }^{5}$ Genome Science Laboratory, RIKEN, Hirosawa, Wako, Saitama 351-0198, Japan
}

\begin{abstract}
The cell cycle is one of the most fundamental processes within a cell. Phase-dependent expression and cell-cycle checkpoints require a high level of control. A large number of genes with varying functions and modes of action are responsible for this biology. In a targeted exploration of the FANTOM2-Variable Protein Set, a number of mouse homologs to known cell-cycle regulators as well as novel members of cell-cycle families were identified. Focusing on two prototype cell-cycle families, the cyclins and the NIMA-related kinases (NEKs), we believe we have identified all of the mouse members of these families, 24 cyclins and 10 NEKs, and mapped them to ENSEMBL transcripts. To attempt to globally identify all potential cell cycle-related genes within mouse, the MGl (Mouse Genome Database) assignments for the RIKEN Representative Set (RPS) and the results from two homology-based queries were merged. We identified 1415 genes with possible cell-cycle roles, and 1758 potential paralogs. We comment on the genes identified in this screen and evaluate the merits of each approach.
\end{abstract}

[Supplemental material is available online at www.genome.org.]

The cell cycle, the process by which a cell replicates itself, is a highly controlled process employing many regulatory mechanisms. The importance of strict cell-cycle control is apparent when disregulation occurs, as it does it in cancer. Medically, the study of cell-cycle genes is of interest for understanding the nature of a given tumor type, but perhaps more tangibly it offers potential targets for chemotherapy (Sampath and Plunkett 2001; Carnero 2002). The recently launched Cancer Molecular Analysis Project (CMAP) at the U.S. National Cancer Institute (NCI) lists cell cycle as the major CMAP ontology for their molecular targets (http://cmap.nci.nih.gov/ Ontologies).

The recently completed mouse genome (Waterston et al. 2002) coupled with the RIKEN-Functional Annotation of Mouse FANTOM transcriptome project (Okazaki et al. 2002), has provided a huge amount of genetic information to the researcher. Assigning function to these genetic elements is the next great step. Similarity to genes of known function is being used to suggest roles for novel proteins. Similarity measures include protein sequence homology, domain-based predictions (Apweiler et al. 2001; http://www.ebi.ac.uk/interpro, The InterPro homepage), and structure-based predictions (Murzin et al. 1995; http://scop.mrc-lmb.cam.ac.uk/scop, Structural Classification of Proteins homepage).

These approaches suggest a function by similarity to another protein; however, they do not present the researcher with a global view of the biology, nor do they present the function within a structured syntax. The Gene Ontology pro-

${ }^{6}$ Takahiro Arakawa, Piero Carninci, Jun Kawai, and Yoshihide Hayashizaki.

${ }^{7}$ Corresponding author.

E-MAIL a.forrest@imb.uq.edu.au; FAX 61-7-3365-4388.

Article and publication are at http://www.genome.org/cgi/doi/10.1101/ gr.1012403. posed by the Gene Ontology Consortium (2001) attempts to assign proteins to a number of structured ontologies. The toplevel hierarchy is split into three ontologies: biological process, molecular function, and cellular component. These ontologies are then divided into lower-level ontologies. Any given gene product can belong to one or many ontologies, dependent upon what is currently known of the protein.

In this paper we attempt to identify genes with a role in the cell cycle, corresponding to biological process gene ontology GO:0007049 (and sub-branches). Within the context of the three higher-level ontologies, cell cycle-related genes encompass many lower-level ontologies. Within the context of cellular component, cell-cycle proteins have many different subcellular distributions, which often change during the course of the cell cycle. Within the context of biological process, cell-cycle genes encompass DNA replication, conformation and integrity, cytoskeletal changes, organelle distribution and reassembly, and most importantly, cell-cycle controllers that orchestrate the entire process.

Within the context of molecular function, cell-cycle regulation and mechanics involve many different classes of proteins at different phases throughout the cell cycle. Regulation at the levels of transcription (Whitfield et al. 2002), translation (Groisman et al. 2002; Horton et al. 2002), phosphorylation (Nigg 2001), and targeted proteolysis (Peters 2002) are all used during the cell cycle.

In the first part of the present study, we used similarity searches directed towards whole proteins and conserved domains to identify novel members of two prototype cell-cycle families, the NIMA-related kinases and the cyclins. Focusing on one family at a time allowed us to evaluate each assignment on a case by case basis. This approach requires good knowledge of each family in question and the level of homology within the given family. Requiring an in-depth knowl- 
edge of each family does not make this approach amenable to a global screen.

In the second part of this study we applied a BLASTPbased approach using a single threshold for all hits. Two sources of cell cycle-related bait sequences were collected to screen the RIKEN variable protein set (VPS). The first set of bait sequences (Bait1) was obtained by downloading sequences corresponding to all the genes assigned cell-cycle roles within the AMIGO gene ontology browser (http:// www.godatabase.org/cgi-bin/go.cgi). The second set of bait sequences (Bait2) was obtained by using a keyword search for cell cycle-related terms within the high-quality nonredundant protein sequence databases SWALL and the International Protein Index (IPI; EBI; http://srs.ebi.ac.uk). The results from both BLASTP searches are presented and compared to the assignments made by the Mouse Genome Informatics (MGI) group for the RPS sequences (Okazaki et al. 2002).

\section{RESULTS AND DISCUSSION}

\section{NIMA-Related Kinases (NEKs)}

NIMA-related kinases (NEKs) are defined by the prototype mitotic regulator NIMA (never in mitosis A), a protein kinase identified in Aspergillus nidulans. This kinase was identified by its ability to complement the nimA5 temperature-sensitive mutation that causes cells to block in late G2 (Osmani et al. 1987). A number of mammalian homologs have been identi- fied; however, only three have been shown to have cell-cycle roles (Nek2, Ha Kim et al. 2002; Nek9, Roig et al. 2002; Nek11, Noguchi et al. 2002).

Using the sequence retrieval system SRS6 (Etzold and Argos 1993; http://srs.ebi.ac.uk), we used keyword searches to extract all publicly available NIMA-related kinase sequences, and then we used these as bait sequences to blast against the RIKEN VPS. All sequences with significant hits were examined manually and mapped to the mouse genome.

In total we identified 10 NIMA-expressed related kinases (Table 1). All of these were either known to or predicted by ENSEMBL (http://www.ensembl.org/Mus_musculus). mRNA sequences for eight of these had been observed previously in mouse $(1,2,3,4,6,7,8$, and 9/nercc 1$)$. The two others were the mouse homolog of Nek11 and a gene predicted by ENSEMBL (ENSMUSG00000037738) but not previously observed as a transcript; for the rest of this paper, this transcript will be termed Nek12. Nek12 is immediately downstream of Nek3, and seems to represent a gene duplication which is conserved in human (Nek3:ENSG00000136098, Nek12:ENSG00000165396). These appear to represent distinct genes, as no transcripts were identified which included exons from both Nek3 and Nek12.

During the course of mapping the NEKs however, transcripts were identified which were able to bridge the gap between multiple ENSEMBL genes. In the case of Nek9, recently published as Nercc1 (Roig et al. 2002), AJ489828

Table 1. NIMA-Expressed Related Kinases and Their Splice Variants in Mouse

\begin{tabular}{|c|c|c|c|c|c|c|c|}
\hline Symbol $^{a}$ & VPS ID ${ }^{\mathbf{b}}$ & cDNA $^{c}$ & Length $^{d}$ & $\begin{array}{l}\text { Domains } \\
\text { present }^{\mathrm{e}}\end{array}$ & ENSEMBL MOUSE $^{f}$ & Chromosome $^{\mathrm{g}}$ & Position \\
\hline \multirow{4}{*}{ Nek1 } & PC3906.3 & AK034754 & 302 & kinase & ENSMUSG00000031644 & 8 & $60172055-60264003$ \\
\hline & PC3906.2 & AK031330 & 424 & $\begin{array}{l}\text { kinase }+ \\
\text { lysine rich }\end{array}$ & & & \\
\hline & PA3906.0 & S45828 & 774 & kinase & & & \\
\hline & PC3906.1 & AK077047 & 941 & $\begin{array}{l}\text { kinase }+ \\
\text { lysine rich }\end{array}$ & $\begin{array}{l}\text { ENSMUSG00000031644 } \\
\text { ENSMUSG00000037970 }\end{array}$ & $\begin{array}{l}8 \\
8\end{array}$ & $\begin{array}{l}60172055-60264003 \\
60269845-60291714\end{array}$ \\
\hline \multirow[t]{2}{*}{ Nek2 } & PC3907.1 & AK077627 & 366 & kinase & ENSMUSG00000026622 & 1 & 193201229-193212725 \\
\hline & PA3907.0 & U95610 & 443 & kinase & & & \\
\hline \multirow[t]{2}{*}{ Nek3 } & PC6195.1 & NP_035978 & 509 & kinase & ENSMUSG00000031478 & 8 & 20858571-20894987 \\
\hline & PA6195.0 & AF099066 & 511 & kinase & & & \\
\hline \multirow[t]{2}{*}{ Nek4 } & PC6196.1 & AK078809 & 744 & kinase & ENSMUSG00000021918 & 14 & 26049834-26085981 \\
\hline & PB6196.0 & NM_011849 & 792 & kinase & & & \\
\hline \multirow[t]{2}{*}{ Nek6 } & PC7896.3 & AK029266 & 305 & kinase & ENSMUSG00000026749 & 2 & 38989294-39024394 \\
\hline & PB7896.0 & NM_021606 & 313 & kinase & & & \\
\hline \multirow[t]{2}{*}{ Nek7 } & PC7895.1 & АК078639 & 250 & kinase & ENSMUSG00000026393 & 1 & 13936116-139440087 \\
\hline & PB7895.0 & NM_021605 & 302 & kinase & & & \\
\hline \multirow[t]{2}{*}{ Nek8 } & PC2055.1 & AK014546 & 291 & kinase & ENSMUSG00000017405 & 11 & $78804211-78814753$ \\
\hline & PB2055.0 & NM_080849 & 698 & $\begin{array}{c}\text { kinase }+ \\
\text { Rcc1 }\end{array}$ & & & \\
\hline \multirow{4}{*}{$\begin{array}{l}\text { Nek9/ } \\
\text { Nercc1 }\end{array}$} & PC30616.0 & BC024926 & 196 & none & ENSMUSG00000021249 & 12 & $80001495-80005923$ \\
\hline & PC30616.X & A) 489828 & 984 & kinase + & ENSMUSG00000021249 & 12 & $80001495-80005923$ \\
\hline & & & & Rcc1 & ENSMUSG00000034290 & 12 & 80007619-80020593 \\
\hline & & & & & ENSMUSG00000034284 & 12 & $80025106-80036284$ \\
\hline \multirow[t]{2}{*}{ Nek11 } & PC22564.1 & AK030186 & 454 & kinase & ENSMUSG00000035032 & 9 & $106012522-106082223$ \\
\hline & PC22564.0 & AK030042 & 628 & kinase & & & \\
\hline \multirow{2}{*}{$\begin{array}{l}\text { Novel- } \\
\text { Nek12 }\end{array}$} & PC23442.1 & AK054168 & 336 & kinase & ENSMUSG00000037738 & 8 & 20838052-20850939 \\
\hline & PC23442.0 & AK032672 & 614 & kinase & & & \\
\hline
\end{tabular}

${ }^{\mathrm{a} G e n e ~ s y m b o l ~ f r o m ~ M G I . ~}$

bVariable Protein Set ID.

${ }^{\circ}$ CDNA accession numbers.

dLength of peptide in amino acids.

eInterPro domains.

${ }^{f} E N S E M B L$ mouse gene assignment.

${ }^{\mathrm{g}} \mathrm{Genomic}$ location. 
bridges three ENSEMBL mouse genes (ENSMUSG21249, ENSMUSG00000034290, ENSMUSG00000034284). In another example, a longer form of Nek1, detected by RIKEN clone 4932438I04, bridges two ENSEMBL genes, ENSMUSG00000031644 and ENSMUSG00000037970.

Interestingly, a number of the NEK genes appear to encode long and short forms (Table 1). The short forms contain the kinase domain, and longer forms may contain extra domains, as is the case with the Rcc1 domain in Nek8 and Nek9. Splice variants for both Nek11 (Noguchi et al. 2002) and Nek2 have been previously identified, and in the latter example were shown to exhibit different activities and expression patterns (Hames and Fry 2002).

\section{Cyclins}

Cyclins are the regulatory subunit of cyclin/cyclin-dependent kinase (CDK) complexes; these are the prime movers of the cell cycle. Specific subsets of these complexes are required during different phases, the best known example being cyclin B/CDC2 (CDK1), required during G2/M progression (Smits and Medema 2001).

In a strategy similar to that used for the NIMA-related kinases, we extracted bait cyclin sequences from the SWALL protein database using SRS6. In the case of the cyclins, we were able to query the database for sequences containing Interpro (Apweiler et al. 2001) domains associated with cyclins, cyclin (IPR004366), or cyclin c-term (IPR004367). It was also possible to directly query the FANTOM2 data for the presence of these domains.

During the course of the analysis, 38 potential cyclins were identified within the RIKEN VPS, using a combination of the domain-based querying and iterative blasts. Upon further analysis, 24 of these held up as known or likely cyclins (Table 2 ). Sequences that failed to carry on to further analysis include three members of the Rb family (p105, p107, p130), three members of the TFIIB family, a number of other unrelated cyclin box containing proteins, and CABLES, a CDK5 interactor.

The 24 cyclins include the known cyclins A1, A2, B1, B2, B3, C, D1, D2, D3, E1, E2, F, G1, G2, H, I, K, T1, T2, and ania-6a (L) and ania-6b. Also included is uracil-DNA glycosylase 2, noted as a cyclin by a number of workers (Murray and Marks 2001). Two potentially novel cyclins were identified, one most closely related to CYCJ, cyclin J of Drosophila (Finley et al. 1996), and the other related to the L-type cyclins ania-6a and $-6 \mathrm{~b}$. Both of these have been observed in the EST evidence. The cyclin J homolog (AK052506) has been seen in human BA690P14.1/FLJ10895, and the novel cyclin L (AK007413) is represented by another EST in mouse (BC027022). A tree showing the relationship between these two novel cyclins and the known cyclins is shown in Figure 1.

Cyclin J was identified in Drosophila in a screen for DmCdc2 (cdk) interactors (Finley et al. 1996). This cyclin has been shown to associate with cdk2 and play a possible role in the nuclear form of the cell cycle that occurs within a common cytoplasm (syncytium), in Drosophila early embryogenesis (Kolonin and Finley 2000). EST evidence suggests that the cyclin $\mathrm{J}$ homolog is mainly expressed in spleen.

Cyclin ania-6a, also referred to as cyclin L, was identified using a differential display screen in rat brain. Dopamine and glutamate have been shown to induce distinct splice forms of ania-6a, and these forms are able to associate with the orphan CDK, PITSLRE. A close relative was also identified in a database search, termed ania-6b (Berke et al. 2001).

All three L-type cyclins have distinct EST distributions.
Although ania-6a and -6b are both reported as brain-specific, there a number of ESTs which indicate that they are expressed in tumors, especially mammary tumors. Ania-6a is also enriched in kidney, whereas ania-6b is enriched in retina. The novel L-type cyclin is represented by ESTs in thymus and bladder.

Another interesting observation when examining the cyclins was the identification of a number of pseudogenes. During the course of annotation and at the BLAST query stage, multiple copies of cyclins B1, B2, D3, L and the novel L were identified within the VPS. In some cases this may be an artefact of the clustering used; however, for two sequences it is clear that they represent processed pseudogenes of cyclins B1 and $\mathrm{B} 2$, in that they correspond to intronless versions of the $\mathrm{B} 1$ and $\mathrm{B} 2$ coding sequence (CDS) with frameshifts. Where it was not possible to discriminate between the two VPS sequences based upon their alignment against the mouse genome, the respective gene was assigned both VPS identifiers. The B1 pseudogene (2610510C05RIK) is transcribed in the sense orientation, maps to chromosome 14 (36962563698151), and has four frameshifts relative to the cyclin B1 CDS. The B2 pseudogene (AK048139) maps to chromosome 8 (71302891-71304001), is transcribed in the antisense orientation, and contains multiple frameshifts relative to cyclin B2 CDS. The degraded CDS is more similar to cyclin B2 from golden hamster (CYCLIN B2 [Mesocricetus auratus] - P37883), which perhaps suggests that this is an older pseudogene.

Early mapping studies identified 10 cyclin B-related sequences within the mouse genome (Lock et al. 1992) In the present study, we identified five which are transcribed, three containing functional CDS, and two transcribed processed pseudogenes (both of which have supporting EST evidence other than RIKEN). Other workers have identified pseudogenes of cyclins D2, D3, G1, and UNG (Xiong et al. 1992; Lund et al. 1996; Kimura et al. 1997), with a transcribed cyclin D2 pseudogene suggested as a marker for decreased ovarian function (Kimura et al. 1997). The presence of processed pseudogenes of the cyclins within the transcriptome raises questions about whether these noncoding RNAs play some role in cyclin regulation.

\section{Large-Scale Identification of Candidate Cell Cycle-Related Genes}

As outlined in the introduction and Methods section, two sets of bait sequences were produced to mine the VPS for cell cycle-related genes. The first set, "BAIT1" contained 1859 sequences that correspond to genes assigned a cell-cycle role by the Gene Ontology Consortium (2001). The second, "BAIT2" contained 4437 sequences which correspond to eukaryotic sequence entries within SWALL and the IPI which have a reference to cell cycle. These bait sequences were formatted as databases and then used in a BLAST query of the FANTOM2 VPS set.

From the BLAST queries, significant hits (e-30) were observed for 698 of the BAIT1 sequences and 1042 of the BAIT2 sequences. If we cross-compare the VPS entries for which a hit was observed, we find that 416 of the VPS hits are shared between the two queries (Fig. 2A). If we then overlay this with the predictions from the MGI (while only considering the higher-quality predictions, that is, those supported by evidence other than SCOP or InterPro), we find 158 VPS entries supported by all three predictions, 495 by two or more predictions, and 1415 predicted by at least one method. These sequences represent the best hits for each query sequence and are those most likely to be homologs of known cell-cycle genes.

If we extend our predictions to consider all sequences

\section{Genome Research}




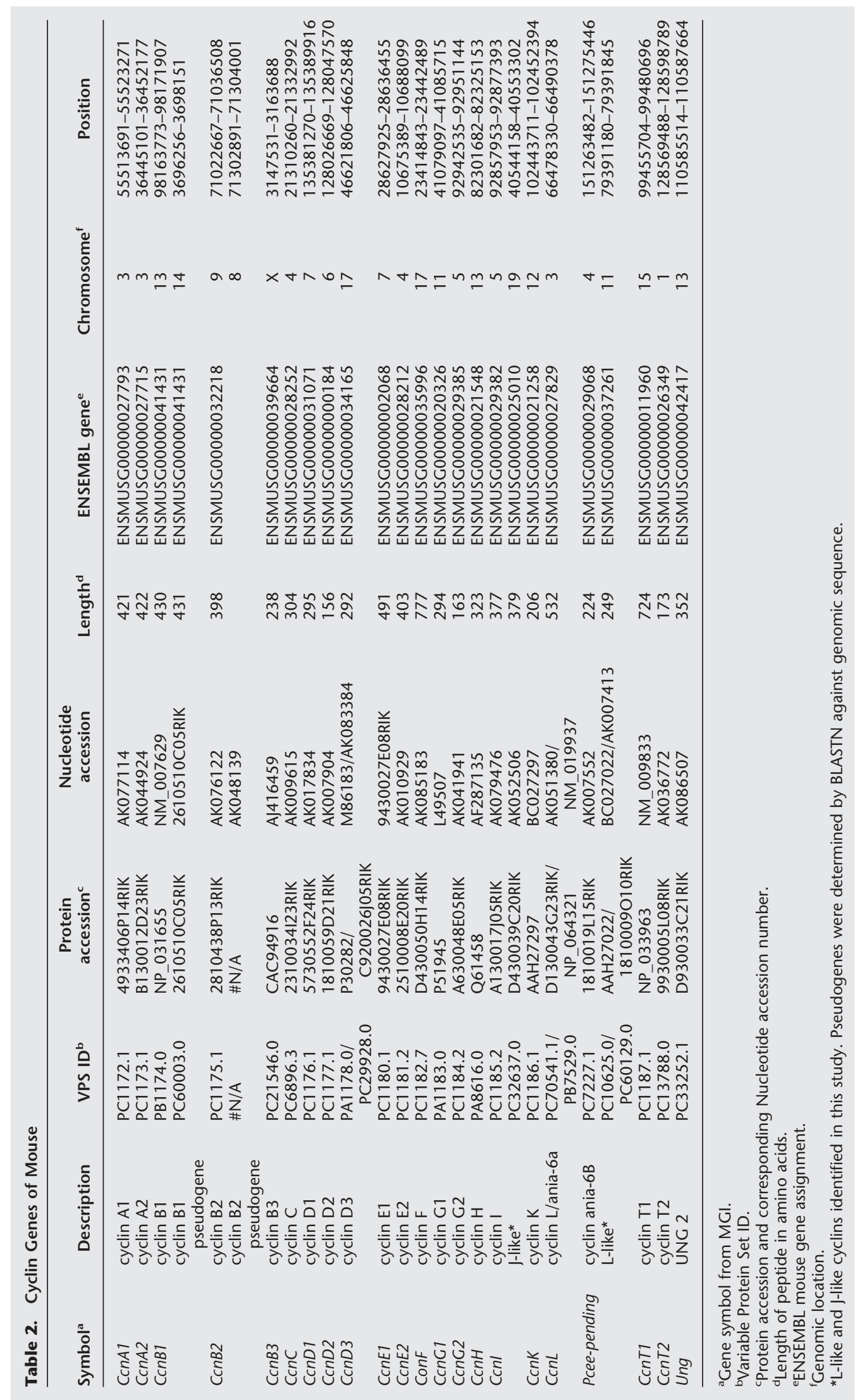


A

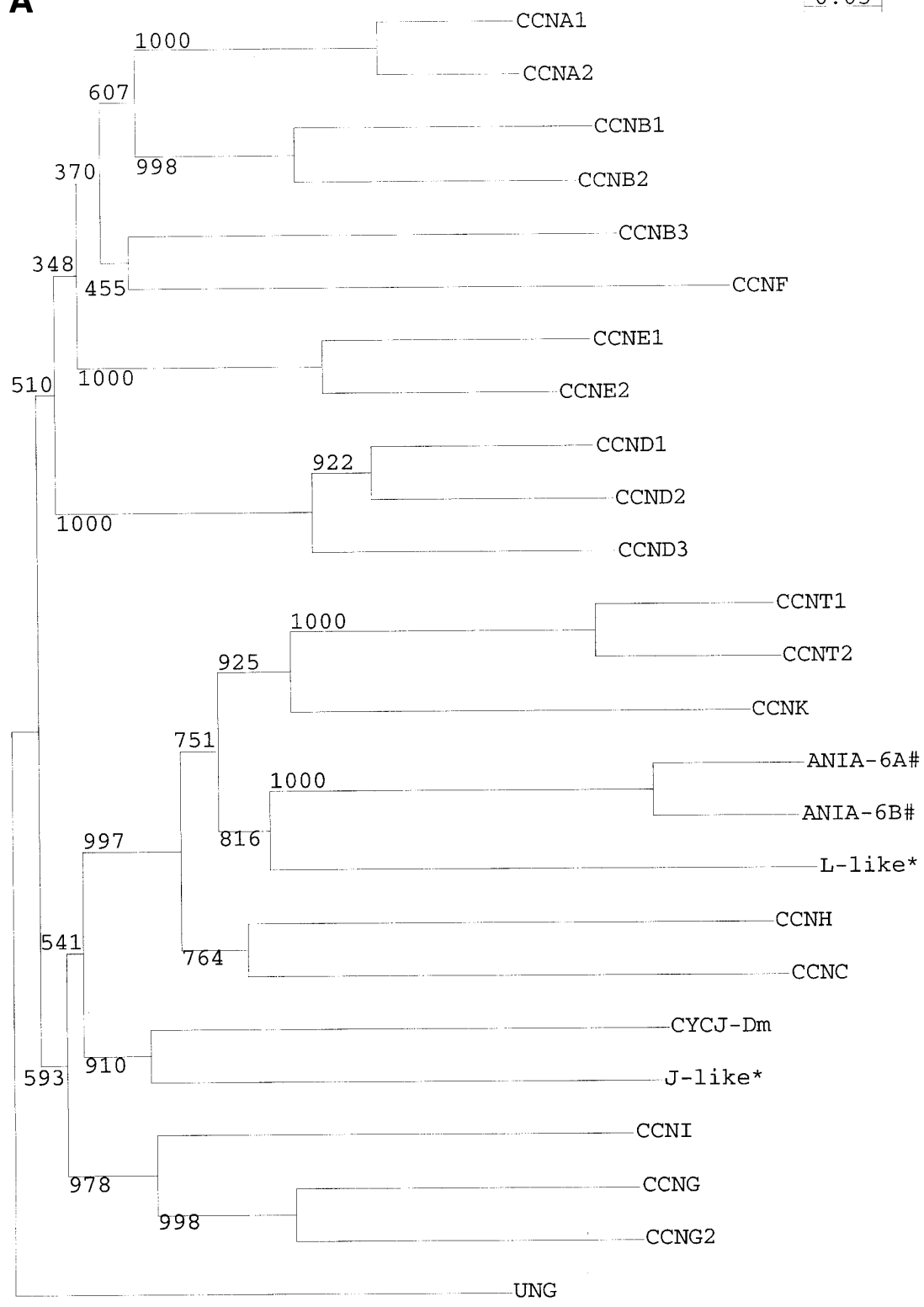

Figure 1 Sequence relationships between cyclins in mouse. (A) Phylogeny of mouse cyclins, based on multiple alignment of the conserved cyclin box domain. Values are shown for 1000 bootstraps. (B) Alignment of the core cyclin box domain of mouse cyclins. Shaded regions and underlying trace show regions of highest sequence similarity. CYCJ-Dm-Drosophila melanogaster cyclin I (AAC47017) is provided as a guide. UNG-Uracil-DNA glycosylase 2 has been recognized as a likely cyclin by a number of workers (Murray and Marks 2001). \#: ANIA$6 \mathrm{~A}$ has been assigned the symbol CCNL. ANIA$6 \mathrm{~B}$, which is clearly a paralog, has been assigned the symbol Pcee-pending. *-L-like and J-like cyclins identified in the present study.

B

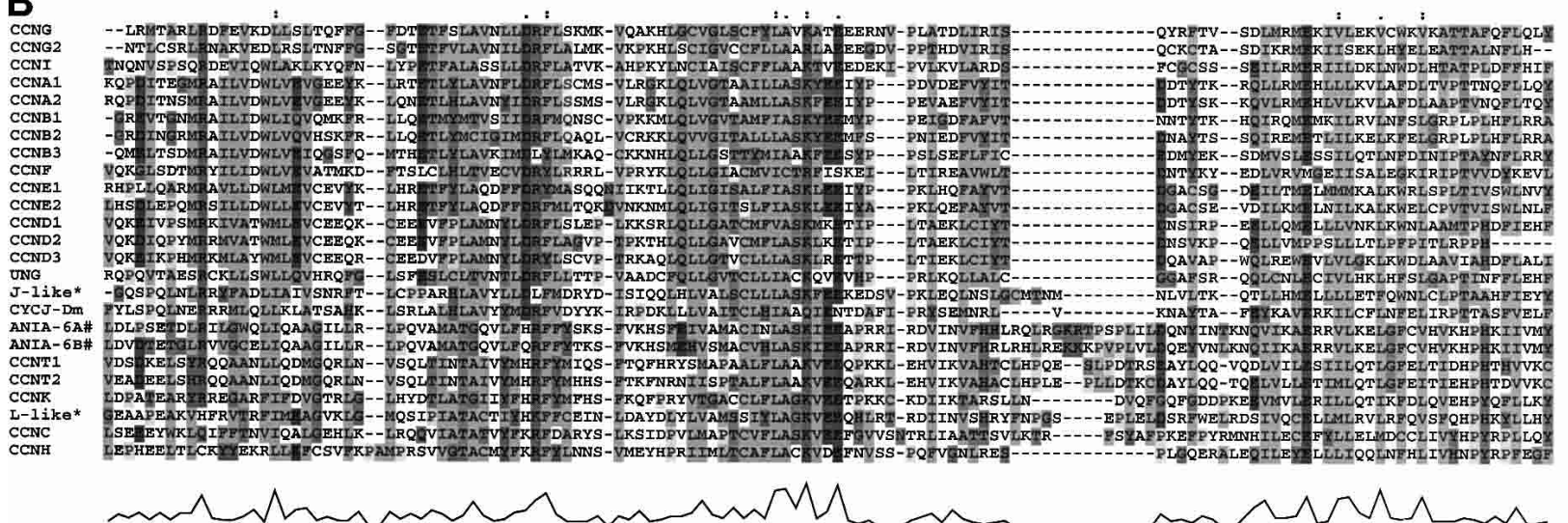


A (1415)

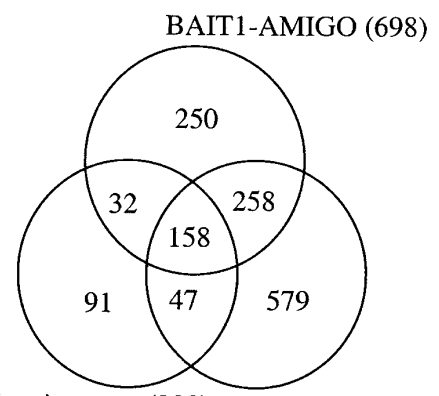

MGI assignment (328) BAIT2-SWALL/IPI (1042)

B (3173)

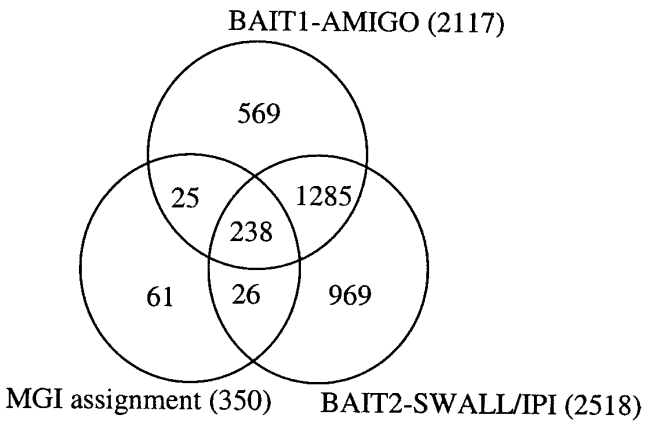

Figure 2 Distribution of candidate cell-cycle genes identified in the global screen. (A) Here, 1415 nonredundant sequences were identified as likely cell-cycle homologs by combining the best-hit predictions from the two BLAST searches and the MGl assignments. BAIT1AMIGO corresponds to the (698) sequences identified by the bait sequences identified by the Gene Ontology Consortium as cell cyclerelated. BAIT2-SWALL/IPI corresponds to the (1042) sequences identified by the bait sequences identified in a keyword search of SWALL and IPI. The $328 \mathrm{MGl}$ assignments are those with good supporting evidence (InterPro and SCOP predictions were excluded). (B) Here, 3173 nonredundant sequences were identified when all significant hits were considered as well as the InterPro-based MGI assignments.

with a significant hit, such that a given bait sequence can have significant hits from more than one VPS sequence, we are able to identify potential paralogs of the genes identified in the first phase (Fig. 2B). When we consider all significant hits and also include the lower trust InterPro domain to GO mappings from MGI, we identify 3173 sequences which are identified by at least one source. These can be broken into the original 1415 candidate cell-cycle genes and 2468 potential paralogs.

\section{Cell Cycle-Related Sequences Missed by This Approach}

The e-value cutoff used in this study is likely to exclude a number of distantly related sequences. During the MATRICS curation phase of the FANTOM2 project, a number of cellcycle families were investigated in detail. Within the raw FANTOM2 data, nine known histone deacetylases (HDACs) and two novels were identified (AK083724, AK045994); similarly, three known centrins and one novel (AK078275) were identified. These sequences served to evaluate first the RPS and VPS sets and secondly the large-scale BLAST approach used in this study.

All of the centrins identified in the FANTOM2 data were also present in the RPS set; however, two of the HDAC sequences were lost, one a known sequence, and one a novel sequence. The BLAST search identified the remaining known centrins and HDACs; however, none of the novel sequences for these two families were identified. This appears to be be- cause the e-value cutoff was set too high; this would argue in favor of a lower threshold. This highlights the problem of requiring family-specific thresholds when evaluating multiple families. In the case of the novel HDAC (AK045994), an e-value cutoff of e-18 would have hit HDAC_MAIZE (probable histone deacetylase [RPD3 homolog]-Zea mays), and similarly the novel centrin (AK078275) would have been detected with a threshold of e-28, hitting CATR_SCHDU (caltractin [centrin]- Scherffelia dubia).

Reassuringly however, the e-30 threshold used allowed us to identify all of the cyclins and NEKs identified in the family-based screen. All members were identified in the paralog table; however, the best-hit table missed the novel nek12.

Due to the nature of keyword-based sequence extraction and the incomplete nature of the Gene Ontology Database, it is unlikely that we have captured all cell cycle-related genes. For a gene with known cell-cycle biology to be included in either set of bait sequences, either the biology must have been recorded in terms of gene ontology, or the sequence entry for that gene must contain cell cycle-related keywords. There are likely to be sequences that have evidence of a cell-cycle role reported in the literature but do not fit either of these criteria. Capturing such sequences requires a more complex textmining-based strategy that would link sequences to evidence in the literature.

\section{Sequences Falsely Assigned as Cell Cycle-Related}

Two potential sources of false positives in this study arise from (1) the use of keyword searches to extract sequences containing cell cycle-related annotations, and (2) the e-value cutoff used. Keyword searches via SRS are not context-sensitive and consequently, there is a failure to discriminate between "... gene $\mathrm{X}$ is involved in cell cycle", "... gene $\mathrm{X}$ is possibly involved in cell cycle" and "... gene X is NOT involved in cell cycle". Bait sequences for which a significant hit was observed had their annotations checked manually for context. Cases where a keyword could be used in another context such as "purified 'metaphase' chromosomes"-[SWALL:'PYRG_ HUMAN'] or "lipid-water 'interphase'" [SWALL:'GLUC_ BOVIN'] were removed on a case by case basis. By careful manual curation, the impact of this was minimized.

The choice of e-value was set high to reduce false positives. As discussed in the introduction, the choice of an optimal e-value suitable for multiple families is difficult, as the level of homology within different families varies significantly. The homolog assignments took the best hit with a score below e-30. Varying the thresholds used for BAIT1 between e-30, e-50, e-80, and e-100 gave $698,603,501$, and 450 hits, respectively. Similarly, varying the thresholds for BAIT2 resulted in 1042, 923, 786, and 700 hits, respectively. The e-values for every hit are provided in the Supplementary data so that researchers can apply a more stringent cutoff if desired.

The impact or e-value cutoff is more significant when considering paralogs. Large highly conserved families such as the zinc finger proteins and protein kinases are overrepresented in the paralogs table. As an example, 192 hits from BAIT2 are from ZF35_MOUSE (zinc finger protein 35), and the next most frequent is 37 hits to FER_HUMAN (protooncogene tyrosine-protein kinase FER). Similarly from BAIT1, 129 hits are from another zinc finger protein, Q9NZH2. If we limit the number of hits to the best hits up to a maximum of five, the numbers for the BAIT2 search drop from 2518 to 1851 and the BAIT1 search from 2117 to 1441.

Another source of overestimation is from bait sequences 
annotated as "similar to cell cycle-related gene X". Similarly, sequences within GO with sequence homology-based evidence may be assigned a cell-cycle role. Thus we end up with a (similar to [similar to cell cycle-related gene X])-type association, where the bait has no direct evidence of a cell-cycle role.

\section{Comparison to Functional Screens}

In a recent microarray-based screen of the human cell line HeLa, 874 genes were identified as cell cycle-regulated (Whitfield et al. 2002). Similarly, in two independent microarraybased screens of budding yeast, 416 and 800 open reading frames (ORFs) of the 6220 monitored transcripts were identified as cell cycle-regulated (Cho et al. 1998; Spellman et al. 1998). As noted by those authors, their work identifies only genes that are regulated at the level of transcription.

The Munich information center for protein sequences (MIPS; Mewes et al. 2002) holds a database of ORF information for $S$. cerevisiae. A simplified gene ontology within MIPS, the 'functional catalog' (http://mips.gsf.de/proj/yeast/ catalogues/) identifies 3936 ORFs with some assigned function, and 451 of these are identified as cell cycle-related. Also within MIPS, the yeast 'phenotype catalog' identifies 1469 ORFs associated with mutant phenotypes, 274 of which are cell cycle-related.

Comparing these estimates of the complement of cellcycle genes within human and yeast, we see an estimated 3\% of human genes and $6.7 \%-18.7 \%$ of yeast genes. The lower estimates are based solely upon microarray data and only identify those genes that are transcriptionally regulated with the cell cycle. The highest estimate of $18.7 \%$ is based on the observed yeast mutant phenotype. In the present study, we identified 1415 sequences as likely cell-cycle proteins; this represents $7.5 \%$ of the 18,768 protein coding genes identified in mouse (Okazaki et al. 2002).

\section{Mouse Cell-Cycle Genes}

Within the best hits there were 512 sequences where the best supporting evidence was from mouse. These included well known cell-cycle genes such as cyclin-dependent kinases (CDK), CDK inhibitors and interactors, cyclins MADs (mitotic arrest-deficient) and BUBs (budding uninhibited by benzimidazoles), cell division controllers (cdc), the E2F transcription factors, histone deacetylases (HDACs), mini-chromosome maintenance (MCMs), calmodulins, septins, RADs, cullins, kinesins, lamins, and a significant number of phosphoregulators such as the mitogen-activated protein kinases (MAPKs), NEKs, Polo-like kinases, CHKs and cdc25 phosphatases, as well as representatives from many other protein families.

Homologs with cell-cycle evidence from 53 other species made up the remaining 903 sequences. Most of these are also known to have cell-cycle roles in mouse; however, as the bait sequence from which the hit was made was not a mouse sequence, it must be inferred that the corresponding mouse entry was not detected by the Gene Ontology Consortium or by the keyword search. The majority of the remaining sequences came from $H$. sapiens (414), S. pombe (149), and $D$. melanogaster (70).

A summary table identifying the candidate cell-cycle sequences is provided as Supplementary Table 1, and includes the evidence for the prediction, and in the case of the BLAST predictions, the e-value and the species of origin, and in the case of the MGI assignments, the evidence and a trust assignment. Lastly, provisional Gene Ontology assignments are provided. Regarding the MGI predictions, these were directly from the MGI assignments. Regarding the sequences from the GO-based BLAST, the assignments were inherited from the bait sequence. The remaining sequences from the keyword search were provisionally assigned gene ontologies based on the keyword used to extract the bait sequence. The predicted paralogs are also presented as Supplementary Table 2.

\section{Definition of a Cell-Cycle Gene}

Evaluating any given gene for a role in the cell cycle based on homolog alone is useful but not completely conclusive. The cyclins demonstrate an important caveat when using homology-based evidence for assigning a role. The ania-6a cyclin clearly meets the definition of a cyclin in that it forms the regulatory subunit of a cyclin/CDK complex with PITSLRE. However, this cyclin has not been shown to have a cell-cycle role (ania-6a; Berke et al. 2001). Similarly, selected members of the 14-3-3 family of proteins have cell-cycle roles (Yaffe 2002), most notably RAD23 and 14-3-3 $\sigma$; however, not all members have been shown to have a cell-cycle role.

In most cases a family can be defined by a motif or shared homology. However, the presence of a domain does not necessarily infer a common biology; there are a number of cyclin box-containing proteins which do not have a cell-cycle role. Historically, families have also been assigned by the phenotype observed. This includes "families" such as the MADs and BUBs (Wassmann and Benezra 2001) and RADs (Rowley 1992) that on a domain basis represent multiple families.

The next point to consider is the definition of a cell cycle-related gene. Cell-cycle controllers are clearly cell cyclerelated, and some of these are expressed in a phase-specific manner; however, the question remains as to whether expression in a phase-dependent manner constitutes a cell-cycle role. Differentiating between these biologies is possible using the structured ontologies presented by the Gene Ontology Consortium (http://www.geneontology.org/). The added benefit of a gene ontology assignment is the evidence code recorded. This gives us some feel of trust in the assignment.

To place a gene within the context of a cell-cycle role based upon homology alone is not enough; predictions need to be verified at the bench. In silico assignments suggest a role for a given gene, but on-bench confirmation is needed to put trust in the assignment. Combined, the in silico predictions guide us when deciding on which experiments to conduct at the bench, and the bench results give us feedback on the in silico model used.

\section{Gene Ontology Associations}

We next assessed the distribution of the various gene ontologies assigned by MGI to the RPS set and looked for associations with the cell-cycle genes identified in Figure 2A. Table 3 shows the top 30 assignments for each of the three top-level hierarchies (molecular function, biological process, and cellular component). For this analysis we ignored the more speculative predictions supported by SCOP.

As expected, cell cycle-related ontologies were enriched in the biological process branch; however, there were a few surprises. The most common ontology within this branch was protein amino acid phosphorylation, followed by regulation of transcription, then regulation of cell cycle. Similarly, within the context of molecular function, transcription factors and protein kinases featured heavily.

Within the context of cellular component, almost a third of the predicted peptides were assigned to the nucleus. The next most common assignments were intracellular location

\section{Genome Research}


Table 3. Top Thirty Gene Ontologies Associated With the Cell-Cycle Genes Identified in Figure 2A

\begin{tabular}{|c|c|c|c|c|c|c|c|c|}
\hline Interpro ID & $\begin{array}{l}\text { Molecular } \\
\text { function }\end{array}$ & Count & Interpro ID & $\begin{array}{c}\text { Biological } \\
\text { process }\end{array}$ & Count & Interpro ID & $\begin{array}{l}\text { Cellular } \\
\text { omponent }\end{array}$ & Count \\
\hline GO:0005524 & ATP binding & 401 & GO:0006468 & $\begin{array}{c}\text { protein amino acid } \\
\text { phosphorylation }\end{array}$ & 170 & GO:0005634 & nucleus & 422 \\
\hline GO:0003677 & DNA binding & 256 & GO:0006355 & $\begin{array}{l}\text { regulation of } \\
\text { transcription, } \\
\text { DNA-dependent }\end{array}$ & 164 & GO:0005622 & intracellular & 133 \\
\hline GO:0016740 & transferase & 192 & GO:0000074 & $\begin{array}{l}\text { regulation of cell } \\
\text { cycle }\end{array}$ & 129 & GO:0016021 & $\begin{array}{l}\text { integral membrane } \\
\text { protein }\end{array}$ & 104 \\
\hline GO:0004672 & protein kinase & 169 & GO:0007049 & cell cycle & 111 & GO:0005615 & extracellular space & 80 \\
\hline GO:0004674 & $\begin{array}{l}\text { protein serine/ } \\
\text { threonine kinase }\end{array}$ & 169 & GO:0008151 & $\begin{array}{l}\text { cell growth and/or } \\
\text { maintenance }\end{array}$ & 95 & GO:0016020 & membrane & 75 \\
\hline GO:0004713 & $\begin{array}{l}\text { protein tyrosine } \\
\text { kinase }\end{array}$ & 158 & GO:0016288 & cytokinesis & 66 & GO:0005737 & cytoplasm & 40 \\
\hline GO:0003700 & transcription factor & 88 & GO:0006281 & DNA repair & 64 & GO:0005856 & cytoskeleton & 35 \\
\hline GO:0016787 & hydrolase & 84 & GO:0006260 & DNA replication & 60 & GO:0005875 & $\begin{array}{l}\text { microtubule } \\
\text { associated } \\
\text { protein }\end{array}$ & 30 \\
\hline GO:0016301 & kinase & 83 & GO:0007242 & $\begin{array}{l}\text { intracellular } \\
\text { signaling } \\
\text { cascade }\end{array}$ & 59 & GO:0005667 & $\begin{array}{l}\text { transcription factor } \\
\text { complex }\end{array}$ & 29 \\
\hline GO:0003676 & nucleic acid binding & 72 & GO:0007165 & signal transduction & 45 & GO:0005694 & chromosome & 21 \\
\hline GO:0005515 & protein binding & 51 & GO:0006810 & transport & 41 & GO:0005739 & mitochondrion & 18 \\
\hline GO:0005525 & GTP binding & 50 & GO:0007017 & $\begin{array}{l}\text { microtubule-based } \\
\text { process }\end{array}$ & 41 & GO:0005886 & plasma membrane & 18 \\
\hline GO:0005509 & calcium ion binding & 48 & GO:0007264 & $\begin{array}{l}\text { small GTPase } \\
\text { mediated signal } \\
\text { transduction }\end{array}$ & 36 & GO:0005871 & kinesin & 17 \\
\hline GO:0003774 & motor & 41 & GO:0007067 & mitosis & 35 & GO:0005783 & $\begin{array}{l}\text { endoplasmic } \\
\text { reticulum }\end{array}$ & 16 \\
\hline GO:0004872 & receptor & 41 & GO:0006508 & $\begin{array}{l}\text { proteolysis and } \\
\text { peptidolysis }\end{array}$ & 33 & GO:0005840 & ribosome & 16 \\
\hline GO:0000166 & nucleotide binding & 40 & GO:0006412 & $\begin{array}{l}\text { protein } \\
\text { biosynthesis }\end{array}$ & 31 & GO:0005718 & nucleosome & 15 \\
\hline GO:0003723 & RNA binding & 39 & GO:0006470 & $\begin{array}{l}\text { protein amino acid } \\
\text { dephosphorylation }\end{array}$ & 27 & GO:0005882 & $\begin{array}{l}\text { intermediate } \\
\text { filament }\end{array}$ & 14 \\
\hline GO:0003685 & DNA repair protein & 33 & GO:0016538 & $\begin{array}{l}\text { cyclin-dependent } \\
\text { protein kinase, } \\
\text { regulator }\end{array}$ & 26 & GO:0005887 & $\begin{array}{l}\text { integral plasma } \\
\text { membrane } \\
\text { protein }\end{array}$ & 14 \\
\hline GO:0003924 & GTPase & 33 & GO:0007275 & development & 24 & GO:0005717 & chromatin & 12 \\
\hline GO:0004386 & helicase & 29 & GO:0007001 & $\begin{array}{l}\text { chromosome } \\
\text { organization and } \\
\text { biogenesis } \\
\text { (sensu Eukarya) }\end{array}$ & 23 & GO:0005794 & Golgi apparatus & 12 \\
\hline GO:0003925 & $\begin{array}{l}\text { small monomeric } \\
\text { GTPase }\end{array}$ & 28 & GO:0008283 & cell proliferation & 23 & GO:0016459 & myosin & 12 \\
\hline GO:0003779 & actin binding & 26 & GO:0006886 & $\begin{array}{l}\text { intracellular protein } \\
\text { transport }\end{array}$ & 22 & GO:0005576 & extracellular & 11 \\
\hline GO:0008026 & $\begin{array}{l}\text { ATP dependent } \\
\text { helicase }\end{array}$ & 26 & GO:0015031 & protein transport & 22 & GO:0005813 & centrosome & 9 \\
\hline GO:0005198 & structural molecule & 25 & GO:0006464 & $\begin{array}{l}\text { protein } \\
\text { modification }\end{array}$ & 21 & GO:0005891 & $\begin{array}{l}\text { voltage-gated } \\
\text { calcium channel } \\
\text { complex }\end{array}$ & 9 \\
\hline GO:0003754 & chaperone & 23 & GO:0008152 & metabolism & 20 & GO:0008287 & $\begin{array}{l}\text { protein } \\
\text { serine/threonine } \\
\text { phosphatase } \\
\text { complex }\end{array}$ & 9 \\
\hline GO:0000158 & $\begin{array}{l}\text { protein phosphatase } \\
\text { type } 2 \mathrm{~A}\end{array}$ & 20 & GO:0006118 & electron transport & 19 & GO:0005663 & $\begin{array}{l}\text { DNA replication } \\
\text { factor } C \text { complex }\end{array}$ & 8 \\
\hline GO:0000163 & $\begin{array}{l}\text { protein phosphatase } \\
\text { type } 1\end{array}$ & 20 & GO:0006334 & $\begin{array}{l}\text { nucleosome } \\
\text { assembly }\end{array}$ & 18 & GO:0005681 & $\begin{array}{l}\text { spliceosome } \\
\text { complex }\end{array}$ & 8 \\
\hline GO:0003777 & microtubule motor & 20 & GO:0006511 & $\begin{array}{l}\text { ubiquitin-dependent } \\
\text { protein } \\
\text { catabolism }\end{array}$ & 18 & GO:0030286 & dynein & 8 \\
\hline GO:0003824 & enzyme & 20 & GO:0006915 & apoptosis & 18 & GO:0005578 & extracellular matrix & 7 \\
\hline GO:0004722 & $\begin{array}{l}\text { protein serine/ } \\
\text { threonine } \\
\text { phosphatase }\end{array}$ & 20 & GO:0007050 & cell cycle arrest & 18 & GO:0005624 & membrane fraction & 7 \\
\hline
\end{tabular}

Gene Ontology assignments from MGI for the RPS sequences were extracted and ranked. Assignments made by SCOP to GO mappings were ignored for this table. 
and integral membrane proteins. Themes that ran through the data set include phosphoregulators (kinases and phosphatases), transcriptional regulation, cytoskeletal proteins, and membrane proteins.

\section{Conclusion}

Using a focused screen of the NIMA-related kinases and the cyclins, we identified all known members and a number of novels. We mapped these sequences to ENSEMBL gene predictions and made comments on their biology.

In the second part of the study we not only identified the majority of mouse genes with known roles in the cell cycle, but also significantly extended the number of cell-cycle assignments. We identified 1415 likely cell-cycle genes and a further 1758 paralogs. Within these two sets we identified novel members of known cell cycle-related families and homologs of cellcycle genes from closely and more distantly related species.

The results of the global screen, the data presented in Supplementary Tables 1 and 2, represent the largest known assignment of cell-cycle genes within mouse and quite possibly within any organism. These entries are indexed by VPS and RPS peptide identifiers. The representative EST accession number, the tentative gene ontology assignments, and the evidence for each of the predictions are also provided. These assignments are provided to the research community as a resource for further investigation and experimental validation.

\section{METHODS}

\section{RIKEN Data Sets Used}

RPS and VPS are nonredundant sequences identified by the RIKEN RTPS group; these attempt to merge all publicly available, high-quality EST sequences with the RIKEN FANTOM2 sequences. The corresponding cDNA and protein sequences are referred to as the RTS (representative transcript set), RPS (representative protein set), and VPS (variable protein set). The VPS aims to produce a set of all unique alternative transcripts from a given transcriptional unit (Okazaki et al. 2002).

For the majority of the present study, the VPS was used. The VPS was used in preference to the RPS to capture cases where a VPS sequence had a better hit with a known cell-cycle regulator than the representative RPS sequence. The raw RIKEN FANTOM2 sequences were accessed using MATRICS (http://fantom2.gsc.riken.go.jp).

\section{Publicly Available Databases Used}

Mouse ENSEMBL cDNA sequences and GENSCAN predictions were downloaded from ENSEMBL (http://www.ensembl.org/ Mus_musculus/). The sequence retrieval system SRS6 (EBI; http://srs.ebi.ac.uk) was used to access SWALL, IPI, and SWISS-PROT sequences. Gene Ontology assignments from the Gene Ontology Consortium (http://www.geneontology.org/) were accessed using the AMIGO gene ontology browser (http://www.godatabase.org/cgi-bin/go.cgi).

\section{Identification of Cyclins and NIMA-Related Kinases (NEKs) Within RPS}

Six hundred forty-two sequences containing the InterPro (Apweiler et al. 2001) motif cyclin (IPR004366) or cyclin c-term (IPR004367) were extracted from SWALL. For the NEKs no such motif exists; in this case, a keyword search was used on SWALL to extract sequences with a NIMA reference. The entries for these candidate NIMA sequences were then inspected manually to determine context, and 30 of these sequences were considered NIMA kinases. Literature searches were carried out to ensure that all known and homologous members were present in the bait sequence database.
In both cases the sequences were formatted as a database and then queried by the VPS. The sequences were ranked by e-value and length of alignment and evaluated on a case by case basis. This allowed us to identify known members, homologs, and novels.

\section{Tree Analysis of Cyclins}

Cyclin sequences were aligned using CLUSTAL V (Higgins et al. 1992). Alignments were edited in a text editor to trim the sequences down to the core cyclin domain, and then reloaded into CLUSTAL V. The sequences were then realigned and used to create a tree using the neighbor-joining method, with 1000 bootstraps. The tree was then visualized and printed using njplot (Perrière and Gouy 1996).

\section{BLAST Searches}

To identify homologous protein sequences, batch BLASTP searches were carried out using BLASTALL (http://www. ncbi.nih.gov/BLAST/). Results were parsed and, except where otherwise mentioned, an expectation value cutoff of e-30 was used to identify significant hits.

To map the cyclins and NEKs to ENSEMBL gene locations, the cDNA sequences corresponding to RPS and VPS sequences were extracted from RTS and compared with BLASTN against the ENSEMBL mouse cDNA sequences. The top three hits for each of the query sequences were examined manually to confirm the hit. This was useful for discriminating between cyclin B1 and its processed pseudogene, and for identifying Nek transcripts that bridged multiple ENSEMBL genes.

\section{Gene Ontology-Based Bait Sequences (BAITI)}

Here, 1767 sequence identifiers were associated with cell cycle-related gene ontologies within AMIGO (http:// www.godatabase.org/cgi-bin/go.cgi ; these were extracted and then used to query SWALL and SWISS-PROT using SRS6 (Etzold and Argos 1993; http://srs.ebi.ac.uk). Sequences corresponding to 1671 of the 1767 gene identifiers were found, sequences for 48 gene symbols could not be located, and 33 of these were the Drosophila Scim genes involved in female meiosis chromosome segregation. These represent mapped loci; however, no sequence is provided (Dobie et al. 2001). From these 1671 identifiers, 1859 sequences were extracted which formed the gene ontology-based bait sequence database (BAIT1).

\section{Keyword Extraction of Cell-Cycle Sequences (BAIT2)}

We used the following cell cycle-related keywords: cdk, cyclin, cell cycle, cdc, cell division, DNA damage, checkpoint, restriction point, mitotic, mitosis, cytokinesis, spindle, kinetochore, prophase, metaphase, anaphase, interphase, meiosis, meiotic, anaphase, prometaphase, telophase, SG2, G2M, MG1, S phase, M phase, G1 phase, and G2 phase, to extract 4437 eukaryotic FASTA sequences from SWALL and the IPI using SRS6. Queries were carried out with individual keywords, for example, Query "([swall-AllText:mitosis])". Entries extracted from each of the individual searches were then merged into the BAIT2 sequence set. The evidence for each bait sequence, in this case, the associated keyword, was kept throughout the entire process to track the ontology suited to each entry. Careful manual curation assured that sequences where the keywords appeared out of context were not included in the BAIT2 sequence set.

\section{Extraction of RPS Sequences Assigned Cell-Cycle Roles by MGI}

Sequences from the RPS project were assigned GO ontologies by the MGI (Okazaki et al. 2002). Any sequence mapping to GO:0007049 or branches thereof were extracted in Microsoft Excel. Note that the MGI assignments were completed on the RPS and not the VPS. VPS is a superset of RPS; consequently, some assignments may have been missed by using the RPS.

\section{Genome Research




\section{ACKNOWLEDGMENTS}

We thank the RIKEN Genome Exploration Research Group Phase I \& II Team, Genomic Sciences Center, RIKEN, and the FANTOM consortium members. The Representative and Variable Protein sets used in these analyses were generated by the RTPS group, and the cell-cycle gene ontology assignments on RPS6 by the MGI Gene Ontology group. We also thank Dr. Rohan Teasdale for challenging discussions on the approach, and Professor David Hume, Institute for Molecular Bioscience, University of Queensland, without whom this collaboration would not have been possible.

\section{REFERENCES}

Apweiler, R., Attwood, T.K., Bairoch, A., Bateman, A., Birney, E., Biswas, M., Bucher, P., Cerutti, L., Corpet, F., Croning, M.D.R., et al. 2001. The InterPro database, an integrated documentation resource for protein families, domains, and functional sites. Nucleic Acids Res. 29: 37-40.

Berke, J.D., Sgambato, V., Zhu, P.P., Lavoie, B., Vincent, M., Krause, M., and Hyman, S.E. 2001. Dopamine and glutamate induce distinct striatal splice forms of Ania-6, an RNA polymerase II-associated cyclin. Neuron 32: 277-287.

Carnero, A. 2002. Targeting the cell cycle for cancer therapy. Br. J. Cancer 87: 129-133.

Cho, R.J., Campbell, M.J., Winzeler, E.A., Steinmetz, L., Conway, A., Wodicka, L., Wolfsberg, T.G., Gabrielian, A.E., Landsman, D., Lockhart, D.J., et al. 1998. A genome-wide transcriptional analysis of the mitotic cell cycle. Mol. Cell 2: 65-73.

Dobie, K.W., Kennedy, C.D., Velasco, V.M., McGrath, T.L., Weko, J., Patterson, R.W., and Karpen, G.H. 2001. Identification of chromosome inheritance modifiers in Drosophila melanogaster. Genetics 157: 1623-1637.

Etzold, T. and Argos, P. 1993. SRS-An indexing and retrieval tool for flat file data libraries. Comput. Appl. Biosci. 9: 49-57.

Finley Jr., R.L. Thomas, B.J., Zipursky, S.L., and Brent, R. 1996. Isolation of Drosophila cyclin D, a protein expressed in the morphogenetic furrow before entry into S phase Proc. Natl. Acad. Sci. 93: 3011-3015.

The Gene Ontology Consortium 2001. Creating the gene ontology resource: Design and implementation. Genome Res. 11: $1425-1433$.

Groisman, I., Jung, M.Y., Sarkissian, M., Cao, Q., and Richter, J.D. 2002. Translational control of the embryonic cell cycle. Cell 109: 473-483.

Ha Kim, Y., Yeol Choi, J., Jeong, Y., Wolgemuth, D.J., and Rhee, K. 2002. NEK2 localizes to multiple sites in mitotic cells, suggesting its involvement in multiple cellular functions during the cell cycle. Biochem. Biophys. Res. Commun.290: 730-736.

Hames, R.S. and Fry, A.M. 2002. Alternative splice variants of the human centrosome kinase Nek2 exhibit distinct patterns of expression in mitosis. Biochem. J. 361: 77-85.

Higgins, D.G., Bleasby, A.J., and Fuchs, R. 1992. CLUSTAL V: Improved software for multiple sequence alignment. Comput. Appl. Biosci. 8: 189-191.

Horton, L.E., Bushell, M., Barth-Baus, D., Tilleray, V.J., Clemens, M.J., and Hensold, J.O. 2002. p53 activation results in rapid dephosphorylation of the eIF4E-binding protein 4E-BP1, inhibition of ribosomal protein S6 kinase, and inhibition of translation initiation. Oncogene 21: 5325-5334.

Kimura, S.H., Kataoka, T.R., Endo, Y., and Nojima, H. 1997. Genomic structure and chromosomal localization of mouse cyclin G1 gene. Genomics 46: 483-486.

Kolonin, M.G. and Finley Jr., R.L. 2000. A role for cyclin J in the rapid nuclear division cycles of early Drosophila embryogenesis. Dev. Biol. 227: 661-672.

Lock, L.F., Pines, J., Hunter, T., Gilbert, D.J., Gopalan, G., Jenkins, N.A., Copeland, N.G., and Donovan, P.J. 1992. A single cyclin A gene and multiple cyclin B1-related sequences are dispersed in the mouse genome. Genomics 13: 415-424.

Lund, H., Eftedal, I., Haug, T., and Krokan, H.E. 1996. Pseudogenes for the human uracil-DNA glycosylase on chromosomes 14 and 16. Biochem. Biophys. Res. Commun. 224: 265-270.

Mewes, H.W., Frishman, D., Güldener, U., Mannhaupt, G., Mayer, K., Mokrejs, M., Morgenstern, B., Münsterkoetter, M., Rudd, S., and Weil, B. 2002. MIPS: A database for genomes and protein sequences. Nucleic Acids Res. 30: 31-34.
Murray, A.W. and Marks, D. 2001. Can sequencing shed light on cell cycling? Nature 409: 844-846.

Murzin, A.G., Brenner, S.E., Hubbard, T., and Chothia, C. 1995. SCOP: A structural classification of proteins database for the investigation of sequences and structures. J. Mol. Biol.

247: 536-540.

Nigg, E.A. 2001. Mitotic kinases as regulators of cell division and its checkpoints. Nat. Rev. Mol. Cell Biol. 2: 21-32.

Noguchi, K., Fukazawa, H., Murakami, Y., and Uehara, Y. 2002. Nek11, a new member of the NIMA family of kinases, involved in DNA replication and genotoxic stress responses. J. Biol. Chem. 277: 39655-39665.

Okazaki, Y., Furuno, M., Kasukawa, T., Adachi, J., Bono, H., Kondo, S., Nikaido, I., Osato, N., Saito, R., Suzuki, H. et al. 2002. Analysis of the mouse transcriptome based on functional annotation of 60,770 full-length cDNAs. Nature 420: 563-573.

Osmani, S.A., May, G.S., and Morris, N.R. 1987. Regulation of the mRNA levels of nimA, a gene required for the G2-M transition in Aspergillus nidulans. J. Cell Biol. 104: 1495-1504.

Perrière, G. and Gouy, M. 1996. WWW-Query: An on-line retrieval system for biological sequence banks. Biochimie 78: 364-369.

Peters, J.M. 2002. The anaphase-promoting complex: Proteolysis in mitosis and beyond. Mol. Cell 9: 931-943.

Roig, J., Mikhailov, A., Belham, C., and Avruch, J. 2002. Nercc1, a mammalian NIMA-family kinase, binds the Ran GTPase and regulates mitotic progression. Genes \& Dev. 16: 1640-1658.

Rowley, R. 1992. Radiation-induced mitotic delay: A genetic characterization in the fission yeast. Radiat. Res. 132: 144-152.

Sampath, D. and Plunkett, W. 2001. Design of new anticancer therapies targeting cell cycle checkpoint pathways. Curr. Opin. Oncol. 13: 484-490.

Smits, V.A. and Medema, R.H. 2001. Checking out the G(2)/M transition. Biochim. Biophys. Acta 1519: 1-12.

Spellman, P.T., Sherlock, G., Zhang, M.Q., Iyer, V.R., Anders, K., Eisen, M.B., Brown, P.O., Botstein, D., and Futcher, B. 1998. Comprehensive identification of cell cycle-regulated genes of the yeast Saccharomyces cerevisiae by microarray hybridization. Mol. Biol. Cell 9: 3273-3297.

Wassmann, K. and Benezra, R. 2001. Mitotic checkpoints: From yeast to cancer. Curr. Biol. 11: 83-90.

Waterston, R.H., Lindblad-Toh, K., Birney, E., Rogers, J., Abril, J.F., Agarwal, P., Agarwala, R., Ainscough, R., Alexandersson, M., An, P., et al. 2002. Initial sequencing and comparative analysis of the mouse genome. Nature 420: 520-562.

Whitfield, M.L., Sherlock, G., Saldanha, A.J., Murray, J.I., Ball, C.A., Alexander, K.E., Matese, J.C., Perou, C.M., Hurt, M.M., Brown, P.O., et al. 2002. Identification of genes periodically expressed in the human cell cycle and their expression in tumors. Mol. Biol. Cell 13: 1977-2000.

Xiong, Y., Menninger, J., Beach, D., and Ward D.C. 1992. Molecular cloning and chromosomal mapping of CCND genes encoding human D-type cyclins. Genomics 13: 575-584.

Yaffe, M.B. 2002. How do 14-3-3 proteins work?-Gatekeeper phosphorylation and the molecular anvil hypothesis. FEBS Lett. 513: $53-57$.

\section{WEB SITE REFERENCES}

http://www.godatabase.org/cgi-bin/go.cgi; AMIGO gene ontology browser.

http://www.ncbi.nih.gov/BLAST; Basic Local Alignment Search Tool homepage.

http://cmap.nci.nih.gov/Ontologies; Cancer Analysis Molecular Project homepage.

http://www.ensembl.org/Mus_musculus; ENSEMBL mouse genome homepage.

http://fantom2.gsc.riken.go.jp; Functional Annotation of Mouse, RIKEN.

http://www.geneontology.org; Gene Ontology Consortium homepage.

http://www.ebi.ac.uk/IPI/IPI; International Protein Index.

http://www.ebi.ac.uk/interpro; InterPro homepage.

http://mips.gsf.de/proj/yeast/catalogues/; MIPS yeast catalogs.

http://www.informatics.jax.org/; MGI 2.8-Mouse Genome Informatics (MGI)

http://srs.ebi.ac.uk; Sequence Retrieval System (SRS6).

http://scop.mrc-lmb.cam.ac.uk/scop; Structural Classification of Proteins homepage.

Received December 3, 2002; accepted in revised form March 31, 2003. 


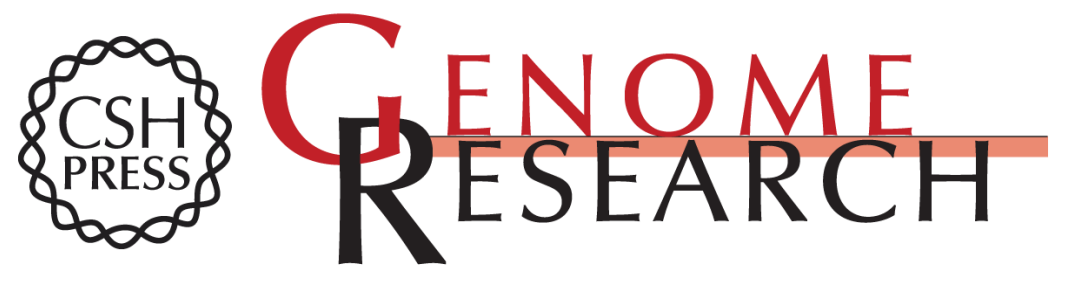

\section{Exploration of the Cell-Cycle Genes Found Within the RIKEN FANTOM2 Data Set}

Alistair R.R. Forrest, Darrin Taylor, RIKEN GER Group, et al.

Genome Res. 2003 13: 1366-1375

Access the most recent version at doi:10.1101/gr.1012403

Supplemental Material

References License

Email Alerting Service
http://genome.cshlp.org/content/suppl/2003/06/22/13.6b.1366.DC1

This article cites 36 articles, 8 of which can be accessed free at: http://genome.cshlp.org/content/13/6b/1366.full.html\#ref-list-1

Receive free email alerts when new articles cite this article - sign up in the box at the top right corner of the article or click here.

\section{Affordable, Accurate Sequencing.}

